
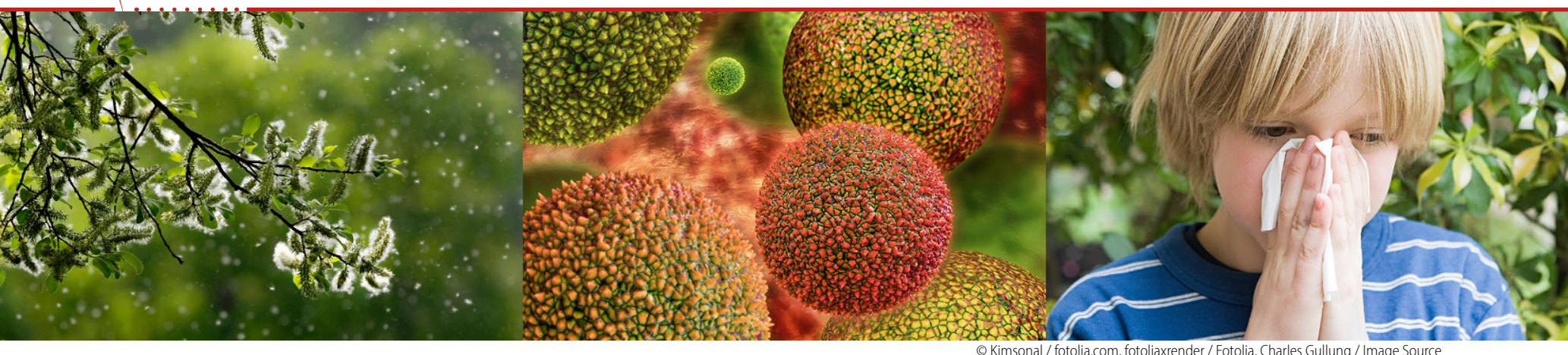

Thomas Ots

Graz, Österreich

\title{
Die Therapie der Pollinosis - Acht Autoren stellen ihr therapeutisches Regime vor
}

\author{
Einleitende Bemerkungen zum Thema
}

\begin{abstract}
Pollinosis in der TCM
Erstaunlicherweise existiert dieses Krankheitsbild nicht in der TCM (Traditionelle Chinessiche Medizin) (siehe hierzu Lehmanns Untersuchung von 35 zwischen 1976 und 1998 erschienenen Akupunkturlehrbüchern in der VR China; [1]). Auch wenn manche chinesischen Bücher die allergische Rhinitis erwähnen, so wird dies nicht auf das Gesamtbild der Pollinosis erweitert, d. h., eine Beteiligung der Augen (Juckreiz, Rötung) wird in diesem Kontext nicht erwähnt.

Die Konjunktivitis wird uneinheitlich beschrieben, zumeist im Kontext der Funktionskreise Leber/Gallenblase und Magen und der pathogenen Faktoren Wind-Hitze.

Die Punkte für Rhinitis in Reihenfolge der Häufigkeit [1]:

- Di 4, 20, Yintang (Ex-KH 3), Lu 7, Gb 20, Bitong (Ex-KH 8), LG 23, Taiyang (Ex-KH 5),

- Di 11, Ma 36, Bl 13, LG 14 ...
\end{abstract}

Deutsche Zeitschrift für Akupunktur

2018 • 61 (1): 16-17

https://doi.org/10.1007/s42212-018-0024-0

(c) Springer Medizin Verlag $\mathrm{GmbH}$, ein Teil von

Springer Nature 2018
Die Punkte für Konjunktivitis in Reihenfolge der Häufigkeit: Di 4, Bl 1, Taiyang (ExKH 5), Gb 20, Le 2, Lu 11, Le 3, LG 23 ...

\section{Westliche Akupunkteure}

Pollinosis wird als eigenständiges Krankheitsbild erwähnt, zumeist aber auf die allergische Rhinitis beschränkt - vor allem in Studien. Da die Therapien im allgemeinen den chinesischen Leitbildern ähneln, wird im Folgenden nur auf bestimmte abweichende Besonderheiten hingewiesen.

Kubiena [2] empfiehlt bei Rhinitis die Kombination von Akupunktur und Homöopathie. Wichtig sei die Unterscheidung zwischen Fülle an Pathogenen und Mangel an Substrat. Bei Fülle schröpft sie Bl 13. Für die Rhinitis allergica gilt prinzipiell dasselbe, zusätzlich Ohrakupunktur (OAP): 78 Allergiepunkt, 55 Shen Men. 13 Nebenniere, 12 Endokrinium.

Bei Konjunktivitis als Basiskombination: Di 4, Bl 1, Gb 1, Le 3, Handpunkt 4 (Augenpunkt). Bei Conjunctivitis allergica: Gb 1, Gb 14, Gb 20, Gb 40, Le 3, Le 8.

Rubach [3] sowie Ogal und Kolster [4] empfehlen bei OAP zusätzlich den Interferon- und Thymuspunkt, vor allem in der prophylaktischen Behandlungsphase.

Bernot [5] empfiehlt auch bei Sinusitis (Wind/Hitze) am Ende der Therapie im Bereich der Nasennebenhöhlen Moxibustion zur Lösung lokaler Blut- und Qi-Stasen.
Bahr und Strittmatter [6] betonen den Allergiepunkt 78 als wichtigsten Punkt bei Heuschnupfen. Punkte gemäß elektrischer Aktivität: Allergie 78 li. in Gold, lokale Punkte, je nach betroffenem Organ: Nase, Augen, Bronchien, Lunge, jeweils betroffene Seite in Gold. Weitere antiallergische/antientzündliche wirksame Punkte: Kortison li, ACTH re., Interferon li., Thymus li., zonendominanter Punkt A li. (alle in Gold). Zur Stärkung der Konstitution Niere und Nullpunkt re., ebenfalls in Gold. Auf Störfelder achten!

Gleditsch [7] kombiniert je nach Irritationsbefund Körper- und Ohrakupunktur mit YNSA, Hand-, Mundakupunktur und Lymph-Belt. Im Akutstadium empfiehlt er Gb 20 bei starkem Pollenflug sowie 3E 7 bei starker Sekretion.

\section{Studienlage Akupunktur}

Hier sei auf die DZA 1/2017 verwiesen: Elf aktuelle Reviews und RCTs zeigen, dass Akupunktur bei allergischer Rhinitis empfohlen werden kann. Sie erwies sich als wirkungsvoll und sicher. Siehe auch: http://www.daegfa.de/PatientenPortal/Anwendungsgebiete.Heuschnupfen.aspx.

Einige Studien halten sich in der Beurteilung zurück und fordern weitere, größere Studien (siehe z. B. https://bmccomplementalternmed.biomedcentral.com/ 
articles/10.1186/1472-6882-8-13). Drei ältere Studien seien noch erwähnt:

- Langer H, Hauswald B. Die therapeutische Wirkung der Akupunktur und Laserpunktur bei Patienten mit Rhinopathia pollinosa. Dtsch Ztschr f Akup. 1989;32:109-111

- Die berühmte multizentrische ACUSAR-Studie der Charitè unter der Leitung von Benno Brinkhaus: http://annals.org/article.aspx?articleid $=1583578$

- Joos, Schott, Zou et al. Immunomodulatory effects of acupuncture in the treatment of allergic asthma: a randomized controlled study. J Altern Complement Med. 2000 Dec;6(6):519-25. https://www.ncbi.nlm.nih.gov/pubmed/11152056

Literatur

1. Lehmann HJ (1999) Akupunkturpraxis - Chinesische Standardtherapie mit Relevanzkarten. Urban \& Fischer, München, S139-156

2. Kubiena G (2009) Praxishandbuch Akupunktur. Urban \& Fischer, München, S 294-299

3. Rubach A (2000) Propädeutik der Ohrakupunktur, 2. Aufl. Hippokrates, Stuttgart, S117-118

4. Ogal HP, Kolster BC (2003) Ohrakupunktur für Praktiker. Haug, Stuttgart, S104-111

5. Bernot J (2015) Akupunkturkombinationen. Verlag Systemische Medizin, Kötzting, S62-63
6. Bahr F, Strittmatter B (2010) Das große Buch der Ohrakupunktur. Hippokrates, München, S116-117

7. Gleditsch JM (2002) MAPS - Mikroakupunktsystme. Hippokrates, Stuttgart, S 156-159

Korrespondenzadresse

Dr. med. Dr. phil. Thomas Ots Burgring 8, $8010 \mathrm{Graz}$, Österreich ots@daegfa.de

Interessenkonflikt. T. Ots gibt an, dass kein Interessenkonflikt besteht.

\section{Glückwunsch für die DZA}

Lieber Herr Ots,

für die erfolgreiche Fertigstellung des anzunehmenden 60. Jahrgangs der DZA möchte ich ganz herzlich gratulieren! Es ist immer wieder erfrischend, sich die Inhalte der DZA zu erlesen. Der Verlagswechsel von Elsevier zu Springer bringt sicherlich Einiges an eher aufwändigen Dingen mit sich. Dafür wünsche ich ein gutes Durchhaltevermögen; auf die erste Ausgabe 2018 bin ich schon gespannt. ;) Mit herzlichen Grüßen aus dem bereits verschneiten Hochschwarzwald,

Christian Yehoash

Chefredaktion "Qi - Zeitschrift für Chinesische Medizin"

Kurator, Autor, Redakteur für Chinesische Medizin

Hochfirstweg 22

D-79853 Lenzkirch

fon: $+49(1) 753461454$

yehoash@zhaohun.de

www.zhaohun.de 